\title{
THE RENAL TUBULAR RESPONSE TO AMINO ACID LOADING ${ }^{1}$
}

\author{
By PAUL D. DOOLAN,² HAROLD A. HARPER, ${ }^{3}$ MAXINE E. HUTCHIN, AND \\ EDWARD L. ALPEN *
}

\author{
(From the Metabolic Research Facility, U. S. Naval Hospital, Oakland, Calif.)
}

(Submitted for publication February 1, 1956; accepted April 24, 1956)

In previous studies from this laboratory (1) it was shown that renal tubular reabsorption of filtered amino acids was virtually complete in the fasting state. However, when a solution of amino acids was administered intravenously and the load filtered by the kidney thus increased, both the amounts of amino acids reabsorbed and those excreted were increased. In quantitative terms the more noteworthy event was the increase in amount reabsorbed. Considerable selectivity was exhibited in the reabsorption of individual amino acids and evidence was obtained which was interpreted as indicating a "competition" for reabsorption between cystine and one or more other amino acids.

In the present studies the solution of amino acids used for loading was administered at a faster rate than in the previously reported experiments and it was supplemented with additional glycine to test the effects of a further increase in the filtered load of amino acids. In separate studies glycine was administered alone and, in terms of amounts reabsorbed and excreted, the results were compared with those obtained when glycine was given as part of the amino acid mixture and comparable filtered loads were achieved.

\section{METHODS}

Three normal subjects-DNW (age 21, ht. $184.2 \mathrm{~cm}$., wt. $85.5 \mathrm{~kg}$.), WRR (age 28, ht. $175.3 \mathrm{~cm}$., wt. $95.0 \mathrm{~kg}$.), and WS (age 29, ht. $168.9 \mathrm{~cm}$., wt. $60.5 \mathrm{~kg}$.) -were given Merck's 10 per cent solution of free amino acids. The experimental protocol is outlined in Table I-A, the rate of infusion being calculated on a basis of its glycine content. The glomerular filtration rate (GFR) was meas-

1 The opinions or assertions contained herein are the private ones of the authors and are not to be construed as official or reflecting the views of the Navy Department or the Naval Service at large.

2 Lieutenant Commander, Medical Corps, United States Navy.

${ }^{8}$ University of California School of Medicine, San Francisco.

4 U. S. Naval Radiological Defense Laboratory, San Francisco. ured by means of inulin clearance and the manner in which the blood and urine specimens were collected was the same as that used in previous studies (1). The designation "high load" is an arbitrary one, meaning not only that the infusion was given at a faster rate but in addition, in the case of the first three experiments, that the amino acid mixture was supplemented with additional glycine.

Three normal subjects-WRR, BVS (age 27, ht. 167.6 cm., wt. $81.7 \mathrm{~kg}$.), and DMS (age 23, ht. $167.6 \mathrm{~cm}$., wt. 95.5 kg.)-received glycine alone, as outlined in Table I-B.

The methods employed for the microbiological assays of the individual amino acids and the exact composition of the amino acid solution used, as determined in this laboratory (lot B-27), have been reported $(1,2)$. The statistical analysis of the data in which each individual's control period measurements were available was made by means of the ' $t$ ' test for paired data. A $p$ value of 0.05 was accepted as the criterion of significance (3).

\section{RESULTS}

\section{Over-all response of the kidney to the infusion of an amino acid mixture}

In Figure 1 the sum of the filtered load of all of the individual amino acids has been plotted against the total amount reabsorbed. The first two points represent mean and grand mean values, respectively, obtained in six experiments. The last point is a grand mean value obtained from the individual high load periods run in three experiments. The same general pattern of renal response noted earlier by us with lower total filtered loads and by other investigators $(4-8)$ is evident; i.e., as the filtered load is progressively increased both the amounts reabsorbed and those excreted are increased. The increase in the amount reabsorbed, however, is not equally proportionate to the increase in that filtered, and as a result the per cent reabsorbed decreases.

\section{Apparent differences in reabsorption patterns among groups of amino acids}

The mean and grand mean amounts of all the amino acids, except cystine, filtered and reab- 
TABLE I

Experimental protocol

\begin{tabular}{|c|c|c|c|}
\hline sensect & $\begin{array}{l}- \\
c o a t r a l \\
223\end{array}$ & 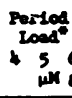 & 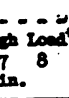 \\
\hline \multicolumn{4}{|c|}{ 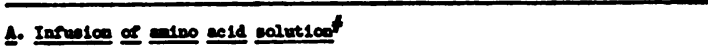 } \\
\hline in & & 25.8 & 39.3 \\
\hline we & & 23.3 & 33.4 \\
\hline $\mathrm{ws}^{+}$ & & 19.9 & 43.2 \\
\hline \multicolumn{4}{|c|}{ 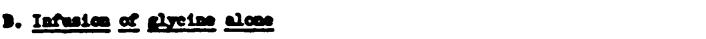 } \\
\hline $\max$ & & 13.2. & \\
\hline 808 & & 27.2 & \\
\hline ms & & 24.4 & $\$ .7$ \\
\hline
\end{tabular}

* A 100-ml. priming dose of the particular solution being studied was administered rapidly when the infusion was started and $15 \mathrm{~min}$. were allowed for equilibration. In subject DMS, a 60-ml. priming dose was used.

\# The supplies of amino acids solution were generously provided by Merck \& Co., Inc.

+ In subject WS the protocol was modified in that 2 load and 3 high load periods were run.

sorbed by the three subjects in the fasting, load, and high load periods, are graphically presented in Figure 2. In the case of the first ten amino acids (reading from the left of the figure), it will be noted that the amounts reabsorbed continued to increase as the filtered load was progressively elevated. This is similar to the overall response made by the kidney to an infusion of an amino acid solution and is what would be predicted for the behavior of the individual amino acids. However, when the load and high load periods are compared in the case of glycine, serine, threonine, and histidine, it will be noted that despite moderate to striking increases in the filtered load the absolute amounts reabsorbed were not significantly different in the high load than in the load periods. In fact, the amount of histidine reabsorbed in the high load period was significantly less than that reabsorbed in the load period. ${ }^{5}$

In Table II the glomerular filtration rate values for all of the subjects and the plasma concentration and excretion values obtained in the case of histidine are listed to provide some information as to the stability of the experimental system as well as to show the increase in histidine excretion.

\footnotetext{
It is not possible, on the basis of the results obtained in these experiments with respect to their reabsorytive mechanism, to state in which group of amino acids tyrosine and glutamine should be included.
}

The decrease which occurred in histidine reabsorption can be calculated from these data.

When the amounts of glycine and histidine reabsorbed in the individual high load periods were analyzed, a tendency to decreased reabsorption in successive periods was observed. This same trend was reflected in the total amount of all amino acids reabsorbed in each period (Figure 3 ) and although in this instance the changes are not statistically significant, the observation is mentioned because of its important implications.

\section{Effect of amino acid loading on the clearance of cystine}

In a previous study (1) it was observed that the clearance of cystine increased during the infusion of an amino acid mixture in the absence of any increase in filtered load of cystine. The infusion mixture is virtually free of cystine (lot B-27 contains $0.09 \mu \mathrm{M}$ per ml.). In Table III there are presented the absolute amounts of cystine filtered and reabsorbed, as well as the clearance of cystine during each of the three periods in the present study. Again it will be noted that the clearance increased during the load period and a further increase was observed in the high load periods. This occurred in the absence of any statistically significant increase in the filtered load of cystine. It is noteworthy that the mean clear-

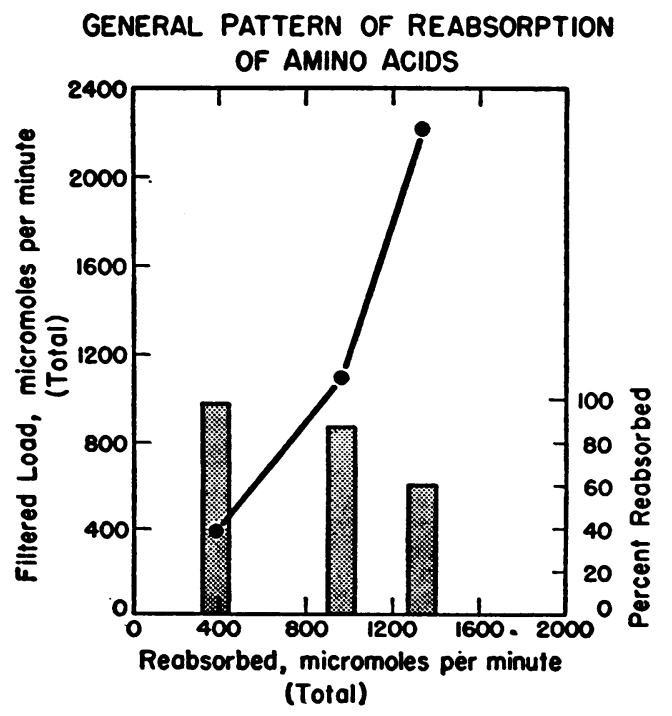

Fig. 1. The General Pattern of Reabsorption of Amrno Acids 

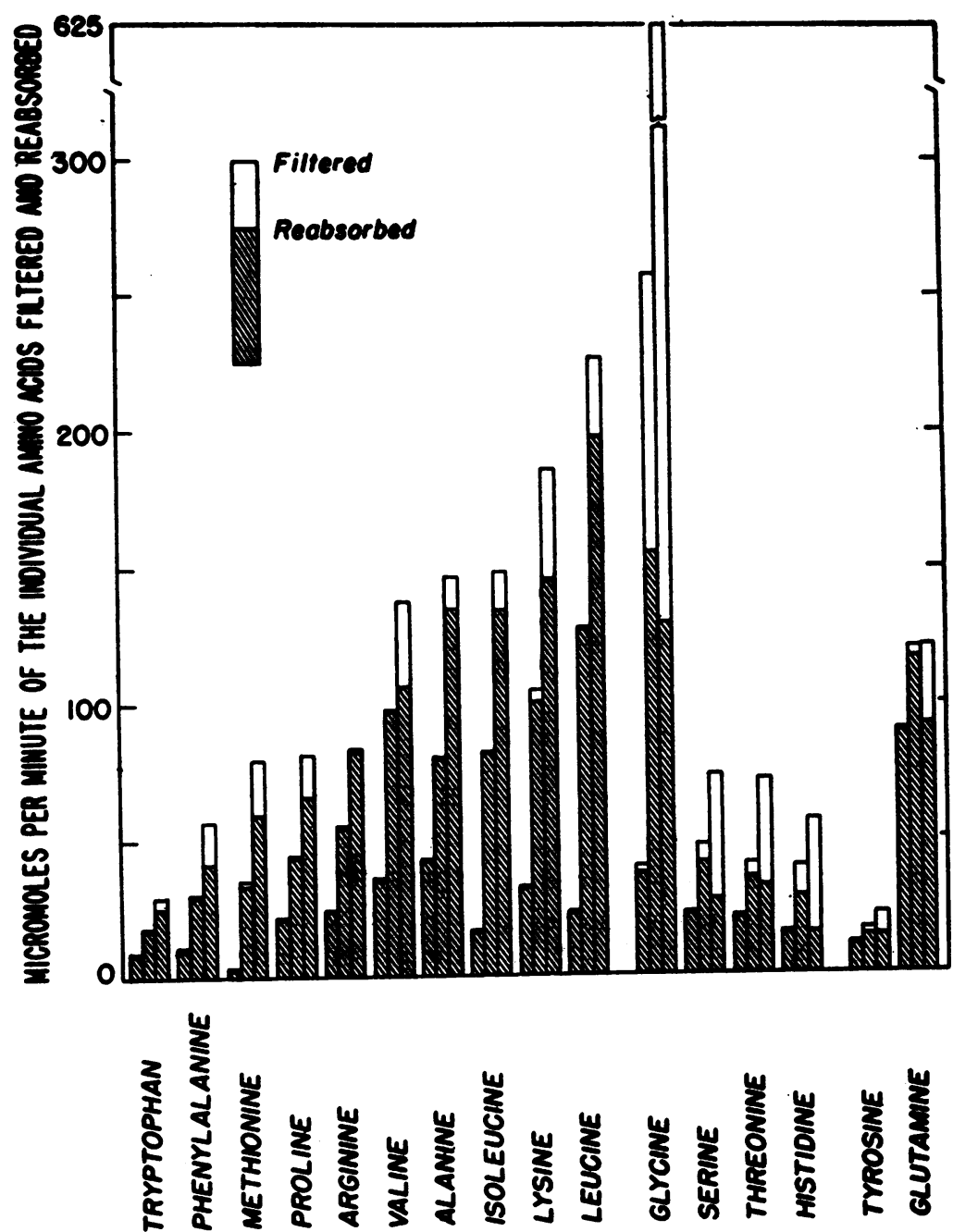

Fig. 2. The Reabsorptive Pattern of the Individual Amino Acids in the fasting (Mean Values), load, and High Load Periods (Grand Mean Values)

TABLE II

Glomerular filtration rate and histidine values in each subject for all periods

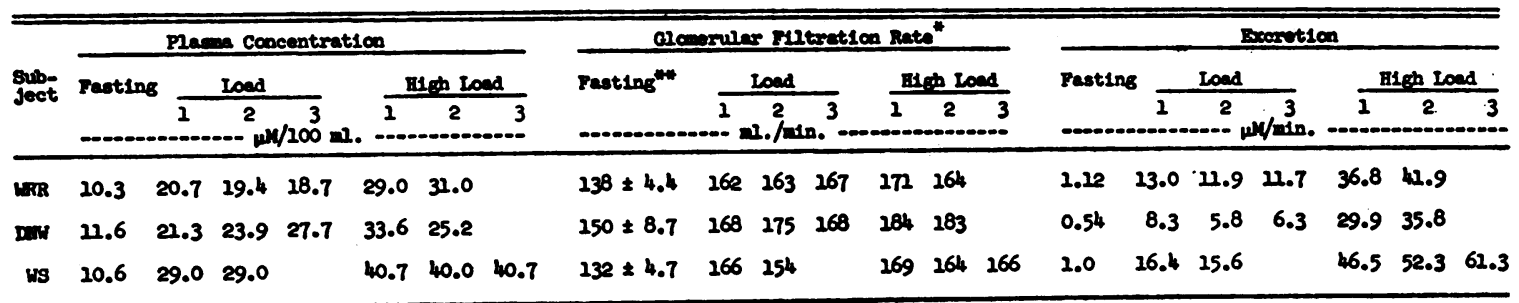

* Uncorrected for surface area. The increase in GFR sometimes noted with these infusions has been commented upon (1).

** Mean value and standard deviations derived from control periods. 
ance (32.6 $\mathrm{ml}$. per min.) achieved during the high load periods approaches 24-hour clearance values observed by us in some patients with cystinuria.

Comparison of the excretion of glycine when infused alone and when incorporated into an amino acid mixture

In Figure 4 the amounts of glycine excreted when it was administered alone are compared with the excretion when it was infused as part of an amino acid mixture and comparable filtered loads achieved. The four columns on the left of the figure represent mean values obtained in four experiments, two wherein glycine was infused alone (subjects WRR and BVS), and two wherein it was part of the amino acid mixture (subjects WRR and WB). In the four columns on the right, the results obtained in a single experiment with glycine alone are compared with those obtained in three experiments wherein the amino acid mixture was infused and comparable filtered loads of glycine achieved. The toxicity of this high dose of pure glycine prohibited further study on other subjects and will be commented on later. Significantly more glycine was excreted when it was infused as part of a mixture and high filtered loads obtained $(p=0.01)$ and although the results obtained at lower filtered loads are not statistically significant, the tubular response appears to be the same.

During the infusion of the glycine solution the clearances of other amino acids were also measured. In subject BVS the clearances of 17 amino acids were measured. In the other two

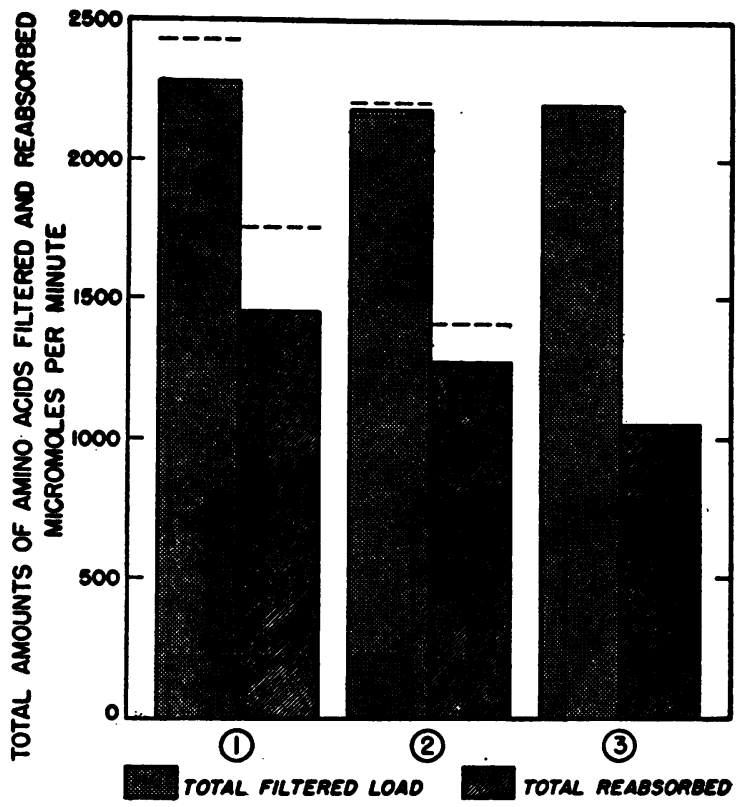

Fig. 3. The Total Amount of All the Amino Acids Filtered and Reabsorbed in Each of the Three High LoAd Periods

The first and second periods are graphed as mean values with the standard deviations (dotted lines) obtained in the three subjects. The third represents the values obtained in a single subject (WS).

experiments (WRR and DMS) only amino acids representative of the types with high (histidine, serine, and threonine) and low (leucine, lysine, and valine) clearance values were measured. The infusion of glycine in all three experiments was accompanied by an increase in the clearances of serine and threonine in addition to that of glycine (Table IV). The plasma concentration of glycine

TABLE III

Cystine-Amounts filtered, reabsorbed, and clearance in each period (mean values)

\begin{tabular}{|c|c|c|c|c|c|c|}
\hline \multirow{2}{*}{ Subject } & \multicolumn{2}{|c|}{$\begin{array}{l}\text { Pasting } \\
\text { Filtered Reabsorbed } \\
\mu \mathrm{M} / \text { min. }\end{array}$} & \multicolumn{2}{|c|}{$\begin{array}{l}\text { lood } \\
\text { Filtered Reabsorbed } \\
\mathrm{H} / \mathrm{min} \text {. }\end{array}$} & \multicolumn{2}{|c|}{$\begin{array}{l}\text { H1Eb Lood } \\
\text { Piltered Reabsorbed } \\
\mu \mathrm{M} / \mathrm{mln} \text {. }\end{array}$} \\
\hline & 16.3 & 16.1 & 27.1 & 16.1 & 25.4 & 14.0 \\
\hline WRR & 13.8 & 13.6 & 16.2 & 15.5 & 16.6 & 22.0 \\
\hline ws & 12.1 & 11.9 & 14.9 & 24.4 & 16.4 & 12.9 \\
\hline \multicolumn{7}{|c|}{$\begin{array}{l}\text { Mean } \\
\begin{array}{c}\text { Mearance } \\
\text { ml./min. }\end{array} \quad 1.92 \pm 0.3^{*} \\
5.91 \pm 1.27^{*}\end{array}$} \\
\hline
\end{tabular}

* Standard deviation. 


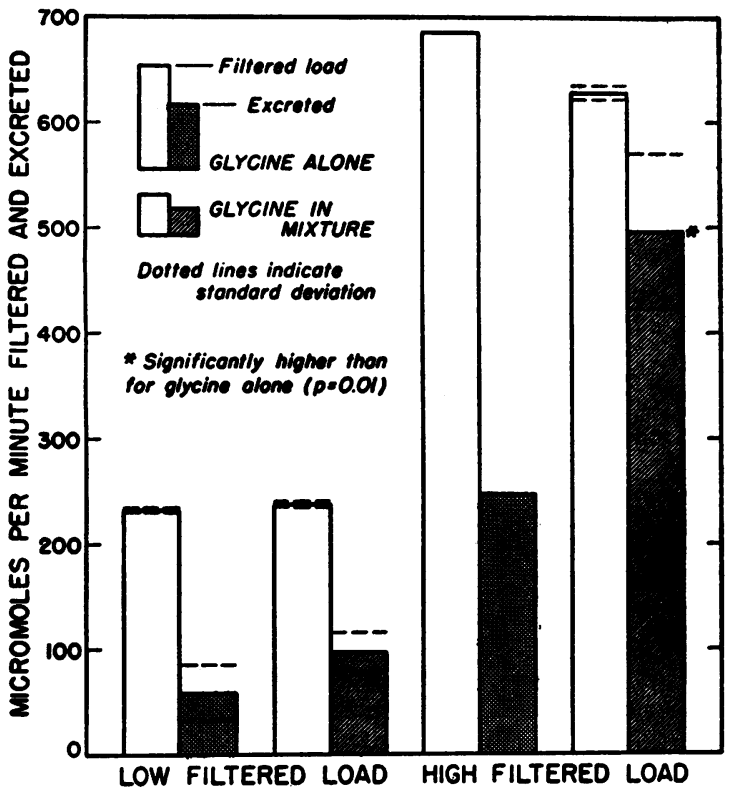

(4)

Fig. 4. Comparison of Amounts Excreted When Glycine Was Infused Alone and As Part of an Amino Acid Mixture

rose in all of the subjects; but in DMS, who received the largest amounts, there was, in addition, a threefold elevation in plasma-serine concentration ( 15.2 to $41.9,47.6$, and $45.7 \mu \mathrm{M}$ per $100 \mathrm{ml}$.) and a twofold elevation in plasma-threonine concentration (14.3 to $28.6,29.4$, and $29.4 \mu \mathrm{M}$ per $100 \mathrm{ml}$.). No changes were observed in plasma concentration or clearance in any of the other amino acids during the glycine infusion.

\section{DISCUSSION}

\section{General features of the amino acid reabsorptive mechanism}

As the filtered load of a single amino acid or of a mixture of amino acids is increased, both the amounts reabsorbed and excreted increase. In this respect the reabsorption of amino acids resembles substances such as urate in man (9) and xylose in the dog (10). Reabsorption itself most certainly involves "active transport" and as such requires energizing reactions. However, the specific reactions concerned and the relation they bear to the rate of transport have not yet been described. The present studies have shown that striking dif-

ferences in rates of reabsorption exist among the individual amino acids, a fact which may be explained by assuming that they are handled by independent mechanisms or by the same mechanism at different rates. It is predicted that in the $a b-$ sence of toxic or antimetabolite phenomena the maximal rate of reabsorption for a mixture of amino acids would vary with the qualitative as well as the quantitative characteristics of the particular mixture being infused, "mixture Tm," while the conventional or true tubular Tm would be attained only if the mixture were constituted in such an ideal manner that both the affinity for and the capacity of the transporting system were maximally utilized.

In the instances wherein $\mathrm{Tm}$ values have been demonstrated for individual amino acids in the $\operatorname{dog}(11,12)$, the saturating value has been attained in a gradual manner and undoubtedly a similar gradual approach would be demonstrated for a mixture of amino acids in human subjects. Pitts (11) has explained this reabsorptive pattern within the framework of Shannon's heuristic hypothesis on the law of mass action as applied to the cellular transporting reaction (13). According to Pitts, the limiting reabsorptive maxima are attained gradually because the velocity of decomposition of an amino acid complex (AB) within the tubular cell is relatively rapid in relation to the attainment of equilibrium in the reaction by which it is formed (A, reabsorbed material and B, some stable cellular component, constant in total amount), $\mathrm{A}+\mathrm{B} \rightleftarrows \mathrm{AB} \rightleftarrows \mathrm{B}+\mathrm{A}$. Decomposition of the complex $A B$ delivers the reabsorbed material to the peritubular blood.

However, an alternative explanation for the

TABLE IV

Changes in clearance of other amino acids noted during the infusion of glycine alone

\begin{tabular}{|c|c|c|c|}
\hline \multirow[b]{2}{*}{ Anino Acid } & \multicolumn{3}{|c|}{ Clearance } \\
\hline & $\begin{array}{c}\text { Fasting } \\
\text { Maan }\end{array}$ & m./min. & $\begin{array}{l}\text { Infusion } \\
\text { Grand Moan } \\
-\ldots .\end{array}$ \\
\hline Glycine & $8.8 \pm 4.5$ & & $34.3 \pm 7.5^{*}$ \\
\hline Serine & $1.26 \pm 1.1$ & & $12.6 \pm 3.5^{*}$ \\
\hline Threonine & $0.5 \pm 0.7$ & & $3.1 \pm 1.1^{+}$ \\
\hline
\end{tabular}

$* p=0.01$

$+p=0.05$ 
gradual approach to $\mathrm{Tm}$ is that among the nephron population there exists a considerable variation in functional capacity to reabsorb amino acids. As a result, varying percentages of the population would become saturated at different plasma levels and the level at which any given nephron or group of nephrons is to become saturated would be a function of the glomerular-tubular balance and/or the distribution of enzymatic activity within the tubular cells. The normal situation which obtains with amino acids might conceivably be analogous to that existing in certain cases of renal glycosuria wherein the maximal reabsorptive capacity for glucose is normal despite the fact that glucose is excreted at relatively low plasma levels $(14,15)$. The existence of two distinct groups of nephrons appears to be the most satisfactory way of explaining this normal Tm glucose (15).

In human subjects it is impossible to collect the type of mean data necessary for a definitive evaluation of either of these hypotheses. On the basis of certain indirect evidence, namely, the competition existing among individual amino acids for reabsorption in the normal subject and the fasting and load excretion values for individual amino acids in patients with various types of aminoaciduria, we believe that variation in functional capacity among the nephron population is neither the sole nor the most important determinant of reabsorptive pattern.

\section{Specific features of the reabsorptive mechanisms}

When the results with the individual amino acids were expressed as grand mean values, certain distinct differences in reabsorptive pattern were noted. Tryptophan, valine, proline, phenylalanine, methionine, lysine, leucine, isoleucine, arginine, and alanine continued to be reabsorbed in greater amounts as their filtered loads increased. The reabsorption of glycine, serine, and threonine, on the other hand, was not significantly different in the load and high load periods despite moderate to striking increases in their respective filtered loads. Moreover, in the case of histidine, a significantly smaller amount was reabsorbed in the high load periods. The reabsorptive capacity for the first group of amino acids obviously was not saturated despite the increases in load, corroborating the findings of earlier studies made at lower plasma levels and reaffirming the prediction that whenever these amino acids are present in the urine in abnormally large amounts, it is more likely due to an impairment in tubular reabsorption than to an "overflow" phenomenon. When the data obtained with glycine, histidine, serine, and threonine are interpreted from the standpoint of the grand mean values, the results suggest that under these experimental conditions some one other or group of other amino acids was successfully competing for or interfering with their reabsorptive mechanisms. The results obtained when glycine was infused alone, as well as the evidence accumulated in a number of other studies $(5,11,16,17)$, all indicate that competition for reabsorption takes place among the individual amino acids.

Although competition undoubtedly exists, evidence has been acquired in the present study that another event is occurring simultaneously. When the amounts of glycine and histidine reabsorbed in the individual high load periods were reviewed, a tendency to reabsorb less in successive periods was observed in each of the three subjects. The actual values were not significant in the case of glycine and of borderline significance $(p=0.06)$ for histidine. This same trend was observed in the total of all the amino acids reabsorbed in each individual high load period. In Figure 3 is presented the total for all the amino acids filtered and reabsorbed as mean values for each of the periods and the trend to reabsorb less with time will be noted. The apparent decrease in total amount reabsorbed plus the fact that the decrease was largely but not completely accounted for by the decrease in glycine and histidine reabsorption suggests that a decrease with time, in reabsorptive capacities for glycine, histidine, and perhaps other amino acids had taken place independently of competitive shifts in reabsorption pattern. This decrease may represent a "fatiguing" of the reabsorptive mechanisms due to an excessive concentration of reaction products, an antimetabolite, or perhaps a toxic phenomenon. The untoward reactions (malaise, nausea, and vomiting) associated with these infusions were sufficiently severe as to deem it imprudent to perform further experiments wherein higher plasma levels would be sustained for longer periods. Animal experiments specifically designed to determine whether any decrease 
in reabsorptive capacity occurs with time are currently in progress.

\section{The tubular response to the administration of glycine}

Glycine was administered as a 2.5 and as a 5.0 per cent solution to two and to one normal subjects, respectively. Greater amounts were reabsorbed when it was thus infused than when it was given as part of an amino acid mixture and comparable filtered loads achieved. In all three subjects there was a significant rise in the clearances of serine and threonine as well as glycine. Increases in the excretion of amino acids other than the one being infused have been observed by others. Beyer and his co-workers (16) originally demonstrated the relationships existing in the reabsorption of leucine and isoleucine as well as arginine, histidine, and lysine. In these same studies no significant competition for. reabsorption was demonstrated between glycine and isoleucine, leucine and arginine. Handler, Kamin, and Harris (18), however, observed a definite increase in arginine and a marked increase in methionine, isoleucine, tryptophan, histidine, and threonine excretion when glycine was infused into dogs at a rapid rate $(3.0 \mathrm{mg}$. glycine nitrogen $\mathrm{N}$ per $\mathrm{kg}$. per min.). Glycine was administered at much slower rates in the present studies ( 0.26 to 1.03 mg. $\mathrm{N}$ per kg. per min.) and no significant increase in histidine, leucine, lysine, valine, alanine, arginine, cystine, isoleucine, methionine, phenylalanine, proline, tryptophan, or tyrosine excretion was observed. Kamin and Handler's studies indicated that histidine and threonine reabsorption could be inhibited most easily by the infusion of other amino acids (17).

In DMS, the subject who received the 5.0 per cent solution of glycine, there was a threefold rise in plasma serine and a twofold elevation in plasma threonine concentration. The rise in plasma serine was not surprising in view of the fact that conversion of glycine to serine is a metabolic route for the disposal of glycine, but the rise in plasma concentration of the essential amino acid, threonine, was unexpected and remains unexplained. No decrease in the plasma concentration of other amino acids, such as Hier (19) observed following the oral ingestion of leucine and isoleucine, was observed in this study.

The implications of the results obtained with glycine must be considered in the knowledge that this amino acid is in certain respects unique. It is the only naturally occurring amino acid which lacks an asymmetric carbon atom and therefore is incapable of isomerization; furthermore, its oxidative deamination in liver and kidney is accomplished by a specific enzyme, glycine oxidase, which catalyzes its conversion to glyoxylic acid. For these reasons the findings reported with respect to glycine cannot necessarily be extended to the other amino acids.

\section{The toxicity associated with glycine infusions}

The infusion of 2.5 per cent glycine was accompanied by a feeling of malaise and nausea in both subjects but the reaction was considered mild. The subject (DMS) who received the 5.0 per cent solution experienced a severe reaction. Approximately 45 minutes after the infusion was begun at $0.44 \mathrm{mg}$. $\mathrm{N}$ per $\mathrm{kg}$. per min., he complained of nausea; 75 to $80 \mathrm{~min}$. after the infusion was started and shortly (15 to $20 \mathrm{~min}$.) after the infusion rate had been increased to $1.03 \mathrm{mg}$. $\mathrm{N}$ per $\mathrm{kg}$. per min., he complained of malaise and began to vomit. The patient appeared quite ill by the time the infusion was discontinued at $104 \mathrm{~min}$. The mean infusion rate was $0.67 \mathrm{mg}$. $\mathrm{N}$ per $\mathrm{kg}$. per min. (the priming doses of glycine are included in calculating the above three infusion rates). The reaction was complicated by what appeared to be a pyrogenic reaction, temperature $100.8^{\circ} \mathrm{F}$., shaking chill, and headache. The malaise, marked weakness, intense nausea, and vomiting persisted for about 9 hours post-infusion. A decrease in GFR occurred during the last two collection periods and the glycine data collected during that period have not been included in any of the results discussed above.

Pitts (11) observed a decrease in glomerular filtration rate and renal plasma flow, vomiting, dilatation and fixation of the pupils, weakness, and incoordination in dogs given 2 to 10 per cent solutions of glycine. These reactions were reversible within a few hours. Simultaneous elevation of plasma glucose and glycine produced near fatal reactions with sharp falls in GFR, coma, and 
decerebrate rigidity. In the experiments of Handler, Kamin, and Harris (18), 8 of 12 dogs expired from respiratory failure when given glycine or glycine plus glucose at a rate of about $1.0 \mathrm{mg}$. $\mathrm{N}$ per kg. per min. The time at which death occurred was inversely proportional to the rate of administration so that toxicity best correlated with total dose rather than the duration of infusion. The fatal dose was quite variable, 8 of the dogs succumbing after receiving 360 to $1301 \mathrm{mg}$. N per $\mathrm{kg}$. and 4 surviving after receiving doses ranging from 703 to $1970 \mathrm{mg}$. $\mathrm{N}$ per $\mathrm{kg}$.

The rate at which the infusion is administered may be more important than Handler, Kamin, and Harris' results would imply, for in subject DMS toxicity became much more apparent and severe shortly after the infusion rate was increased to 1.03 mg. $\mathrm{N}$ per $\mathrm{kg}$. per min. The total dose he received was only $69.7 \mathrm{mg}$. $\mathrm{N}$ per $\mathrm{kg}$. If glycine is given at such a rapid rate that the rate of deamination exceeds the rate of removal of ammonia (urea formation), deaths from ammonia intoxication could result. Evidence in support of this explanation has been acquired by the authors in three separate experiments performed on dogs wherein blood ammonia concentrations have been serially determined during the infusion of glycine in lethal quantities. At the time of death the blood ammonia was found to be markedly elevated. These studies are being continued in an attempt to define more completely the mechanisms concerned.

\section{SUMMARY}

An amino acid mixture, supplemented with additional glycine, was administered at a faster rate than employed in earlier studies in order to determine the effect of the additional increase in the total filtered load of amino acids.

As the total filtered load of amino acids was raised to $2200 \mu \mathrm{M}$ per minute, greater quantities were both reabsorbed and excreted.

The amounts of alanine, arginine, isoleucine, leucine, lysine, methionine, phenylalanine, proline, tryptophan, and valine reabsorbed continued to increase as their filtered loads were elevated.

On the other hand the amounts of glycine, serine, and threonine reabsorbed were no greater in the high load than in the load periods and in the case of histidine significantly less reabsorption occurred. A suggested explanation for this latter finding is the simultaneous operation of two factors, competition among individual amino acids for reabsorption and a "fatiguing" of specific transport systems.

Although during the infusion of the amino acid mixture the filtered load of cystine was not changed, its clearance increased to a maximum of $32.6 \mathrm{ml}$. per min., a value aproaching that which we have observed in some patients with cystinuria.

Glycine, when infused alone, was more completely reabsorbed than when incorporated into an amino acid mixture and comparable filtered loads achieved. The infusion of glycine was accompanied by an increase in the excretion of serine and threonine and in one instance by an elevation in plasma concentration of serine and threonine.

The infusion of glycine proved to be toxic and separate data were collected indicating that this may have been due to ammonia intoxication.

\section{REFERENCES}

1. Doolan, P. D., Harper, H. A., Hutchin, M. E., and Shreeve, W. W., Renal clearance of eighteen individual amino acids in human subjects. J. Clin. Invest., 1955, 34, 1247.

2. Harper, H. A., Hutchin, M. E., and Kimmel, J. R., Concentrations of nineteen amino acids in plasma and urine of fasting normal males. Proc. Soc. Exper. Biol. \& Med., 1952, 80, 768.

3. Snedecor, G. W., Statistical Methods Applied to Experiments in Agriculture and Biology. 4th ed., Ames, The Iowa State College Press, 1946.

4. Kirk, E., Studies on the amino acid clearance. Acta med. Scandinav., 1936, 89, 450.

5. Pitts, R. F., A comparison of the renal reabsorptive processes for several amino acids. Am. J. Physiol., 1944, 140, 535.

6. Goettsch, E., Lyttle, J. D., Grim, W. M., and Dunbar, $P$., The renal amino acid clearance in the normal dog. Am. J. Physiol., 1944, 140, 688.

7. Ferguson, F. P., Eaton, A. G., and Ashman, J. S., Renal reabsorption of methionine in normal dogs. Proc. Soc. Exper. Biol. \& Med., 1947, 66, 582.

8. Russo, H. F., Wright, L. D., Skeggs, H. R., Tillson, E. K., and Beyer, K. H., Renal clearance of essential amino acids: Threonine and phenylalanine. Proc. Soc. Exper. Biol. \& Med., 1947, 65, 215.

9. Berliner, R. W., Hilton, J. G., Yü, T. F., and Kennedy, T. J., Jr., The renal mechanism for urate excretion in man. J. Clin. Invest., 1950, 29, 396.

10. Shannon, J. A., The tubular reabsorption of xylose in the normal dog. Am. J. Physiol., 1938, 122, 775. 
11. Pitts, R. F., A renal reabsorptive mechanism in the dog common to glycin and creatine. Am. J. Physiol., 1943, 140, 156.

12. Wright, L. D., Russo, H. F., Skeggs, H. R., Patch, E. A., and Beyer, K. H., The renal clearance of essential amino acids: Arginine, histidine, lysine and methionine. Am. J. Physiol., 1947, 149, 130.

13. Shannon, J. A., Renal tubular excretion. Physiol. Rev., 1939, 19, 63.

14. Bradley, S. E., Bradley, G. P., Tyson, C. J., Curry, J. J., and Blake, W. D., Renal function in renal diseases. Am. J. Med., 1950, 9, 766.

15. Reubi, F. C., Glucose titration in renal glycosuria in Ciba Foundation Symposium on the Kidney. London, J. \& A. Churchill, Ltd., 1954, p. 99.
16. Beyer, K. H., Wright, L. D., Skeggs, H. R., Russo, H. F., and Shaner, G. A., Renal clearance of essential amino acids: Their competition for reabsorption by the renal tubules. Am. J. Physiol., 1947, 151, 202.

17. Kamin, H., and Handler, P., Effect of infusion of single amino acids upon excretion of other amino acids. Am. J. Physiol., 1951, 164, 654.

18. Handler, P., Kamin, H., and Harris, J. S., The metabolism of parenterally administered amino acids. I. Glycine. J. Biol. Chem., 1949, 179, 283.

19. Hier, S. W., Influence of ingestion of single amino acids on the blood level of free amino acids. J. Biol. Chem., 1947, 171, 813. 